\title{
The Influence of Product Aesthetics on Consumer Inference Making
}

\author{
CAMMY CROLIC, YANMEI ZHENG, JOANDREA HOEGG, AND JOSEPH W. ALBA
}

\begin{abstract}
Product aesthetics can enhance consumer welfare in numerous ways. Aside from simply making products more pleasurable, product aesthetics can also influence the inferences that consumers make about functional attributes. In some instances, an attractive design can accurately provide information regarding utility. In other instances, however, an attractive design can be a misleading signal that prompts consumers to assume more utility than justified. Across five studies, the present research examines whether aesthetics can exert an unwarranted influence on the estimation of missing attribute information in favor of an aesthetically superior product. We show that aesthetics can bias consumers' inferences about functionality, sometimes overriding other more diagnostic information. Boundaries to this effect are also identified that may serve to correct the bias and preserve consumer welfare.
\end{abstract}

$\square$ or nearly two decades, a growing body of research has addressed how product aesthetics can influence consumer response (Bloch 1995). At a general level, research has variously shown that attractive design can enhance product satisfaction and liking, impressions of prestige, luxury, and usability, as well as product and firm value (e.g., Hagtvedt and Patrick 2008; Townsend and Shu 2010). At the level of specific aesthetic dimensions, research has similarly found that positive affective and evaluative responses to an offering can be elicited by characteristics such as prototypicality, proportionality, color, symmetry, novelty, coolness, and cuteness (e.g., Veryzer and Hutchinson 1998; Raghubir and Greenleaf 2006; Labrecque and Milne 2012; Mugge and Schoormans 2012; Mugge, Dahl, and Schoormans 2018; Luffarelli, Stamatogiannakis, and Yang 2019; Schnurr 2019; Warren and Reimann 2019).

Despite this evidence, it has been argued that research on product design still lacks a firm foundation (Homburg, Schwemmle, and Kuehnl 2015) and leaves many questions unanswered (Luchs, Swan, and Creusen 2016). An unanswered question of present concern pertains to the effect that design has on consumer well-being, apart from the direct and inherent benefits of aesthetic pleasure. Patrick (2016) has suggested that "everyday aesthetics" can improve life by influencing consumers to make better decisions for themselves and the world. We fully agree but speculate that everyday aesthetics may also have a negative effect on consumer well-being by biasing decision making. Specifically, the present research examines whether aesthetics might seduce consumers into forming inappropriate impressions of some functional aspect of a product. If so, consumers can unknowingly make suboptimal purchase decisions, thereby reducing their welfare.

Most consumer research on aesthetics has focused on the influence of a design feature on global judgments of the object or the relative influence of form and function on overall product evaluation. For example, research on the halo-like properties of aesthetics has shown that an attractive design can affect overall judgments such as product valuation and expected satisfaction (Raghubir and Greenleaf 2006; Townsend and Shu 2010). In the rare instances in which research has examined the effect of design on specific features, it has focused on the evaluation of explicit product attributes or dimensions. Most akin to the present effort, Hoegg, Alba, and Dahl (2010) pitted visual attractiveness against verbally described functionality on a specific attribute that could not be assessed from visual inspection. Hoegg and colleagues found that the perceived superiority of a brand that excelled on a

\footnotetext{
Cammy Crolic (cammy.crolic@sbs.ox.ac.uk) is an associate professor of marketing, Saïd Business School, University of Oxford, Oxford OX1 1HP, United Kingdom. Yanmei Zheng (yanmeiz@hawaii.edu) is an assistant professor of marketing, Shidler College of Business, University of Hawaii, Honolulu, HI 96822. JoAndrea Hoegg (joey.hoegg@sauder.ubc.ca) is the associate professor and chair of the Marketing and Behavioural Science Division and the alumni professor in marketing, Sauder School of Business, University of British Columbia, Vancouver BC V6T 1Z2, Canada. Joseph W. Alba (joe.alba@warrington.ufl .edu) is the James W. Walter Eminent Scholar Chair and Distinguished Professor of Marketing, Warrington College of Business Administration, University of Florida, Gainesville, FL 32611.
}

JACR, volume 4, number 4. Published online July 24, 2019. http://dx.doi.org/10.1086/705033

(C) 2019 the Association for Consumer Research. All rights reserved. 2378-1815/2019/0404-0008\$10.00 
verbally described functional attribute was amplified when the brand was inferior in terms of visual attractiveness. They characterized this outcome as a polarization effect wherein the inferior design of the functionally superior brand violated consumer expectations, resulting in deeper processing of the target attribute.

Unlike Hoegg and colleagues, the present research examines how product aesthetics influence consumer assessment of unstated functional attributes. As we elaborate below, the distinction is important not only because the target of assessment is fundamentally different but also because the inference context allows for multiple and competing bases for making the assessment, including visual design. Although a small body of research has investigated ways in which the appearance of a brand's logo can alter product inferences (e.g., Jiang et al. 2016; Rahinel and Nelson 2016), to our understanding no research has examined how product aesthetics influence the inferences consumers make regarding feature information that is not plainly available. This is an important gap, inasmuch as consumers' inferences about a product's functional features have a self-evident impact on decision quality. Indeed, the ubiquity and importance of inference-making behavior are reflected in periodic reviews required to characterize the sizeable body of research devoted to the topic (e.g., Alba and Hutchinson 1987; Kardes, Posavac, and Cronley 2004; Kardes et al. 2008).

In a typical investigation within this domain, individuals are presented with explicit verbal information about some attributes and are asked to make inferences about other, unstated verbal attributes (Broniarczyk and Alba 1994). Curiously, this extensive literature has not examined product aesthetics as the bases for inference. Thus, a gap in understanding resides at the intersection of the massive literature on inference making and the growing literature on design. Our results fill this gap by showing how aesthetic design influences consumers' inferences differently from other attributes and in ways that have significant implications for decision quality. Specifically, consumers rely on attractiveness as a signal of functional performance, even in the presence of more objective performance-related information.

\section{DIAGNOSTICITY}

The Accessibility Diagnosticity Model (Feldman and Lynch 1988) argues that the cues that consumers use to make a variety of product judgments depend on the accessibility and perceived diagnosticity of those cues. In the present context, accessibility plays a lesser role because consumers are asked to make a stimulus-based judgment, such that the to-be-inferred information is more likely to be constructed on the basis of explicit cues rather than retrieved from memory. Diagnosticity, on the other hand, looms large because inference making is by nature accompanied by risk, and consumers should be inclined to base their inferences on cues they deem most diagnostic of the missing information to minimize risk. Unfortunately, the model provides little explicit guidance regarding cue diagnosticity. As Lynch and colleagues note, diagnosticity is subjective and in need of further investigation to understand its parameters (Feldman and Lynch 1988; Lynch, Marmorstein, and Weigold 1988). We attempt to address this shortfall within the context of inference making and product aesthetics.

A reasonable starting hypothesis is that when a product attribute cannot be discerned from a visual inspection, product appearance should exert little influence on consumers' inferences about the attribute, especially when other more diagnostic information is available. For a variety of reasons noted below, explicit verbal details about other functional attributes often are more diagnostic. Nonetheless, there is reason to examine the competing effects of appearance and these diagnostic cues. It has been argued that aesthetic cues implicitly and automatically generate favorable attributions about the product (Wu et al. 2017). Such responses can be difficult to overcome and thus should bias inferences in favor of an aesthetically superior product. At a more cognitive level, consumers may interpret the quality of design as a signal of the effort made by the firm to create the product (Wu et al. 2017). If no other compelling basis for inference is available, either tendency could serve the consumer well. However, if other more diagnostic cues are present, consumers should suppress their natural inclinations in order to enhance decision quality. Thus, the welfare implications of product aesthetics can only be properly assessed when considering whether alternative bases for inference are present. The present research examines the tendency to be influenced by aesthetics in the face of alternative inputs to inference making.

\section{BASES FOR INFERENCE}

One focus of the extensive literature on inference making revolves around the numerous bases on which people make inferences (Broniarczyk and Alba 1994; Kardes et al. 2004). For example, one attribute can be inferred from another based on the perceived ecological correlation between the attributes. Consumers can also possess a "theory" regarding the relationship between one attribute and another, such as 
when the durability of a product is inferred from the length of its warranty. The present research examines two related bases for inference: evaluative consistency and market efficiency vis-à-vis the use of product aesthetics as the basis for inference.

\section{Evaluative Consistency}

Evaluative consistency entails making an inference based on the overall evaluative implication of the observable attributes. Within a typical product paradigm, consumers are presented with competing brands - one of which is globally superior to the others on the explicitly described attributesand are asked to make an inference about a missing attribute. An evaluative consistency-based inference arises when the brand that excels on the stated attributes is inferred to excel on the missing attribute based on the brand's overall superiority.

We chose to pit the influence of product aesthetics against evaluative consistency for three reasons. First, evaluative consistency is an intuitive, defensible, and common basis for inference that should serve as a strong counterweight to aesthetics. Second, just as aesthetics can exert a global influence on product perception, evaluative consistency inferences are based on a global evaluation of the explicit attributes. Finally, neither inferences based on aesthetics nor inferences based on evaluative consistency require a major cognitive investment, in that both exert a nearly instantaneous, halo-like influence (Kardes et al. 2004). Given prior research demonstrating the powerful influence of aesthetics on judgment, aesthetics may override evaluative consistency, resulting in inferences that are biased in the direction of the more aesthetically appealing product. However, the paucity of research on inference making in the presence of aesthetic cues makes the prediction less than straightforward. In the presence of product performance information that supports an evaluativeconsistency inference, aesthetics may be ignored when inferring a missing functional attribute. In light of these observations, competing hypotheses emerge.

H1A: Aesthetic design will exert an overriding influence on inference formation, relative to the influence of evaluative consistency.

H1B: Evaluative consistency will exert an overriding influence on inference formation, relative to the influence of the aesthetic design.

\section{Market-Based Inferences}

The market offers a number of cues that can help consumers negotiate purchase decisions. Market-efficiency inferences are based on the idea that, in a competitive environment, brands within a category offer equivalent value (Chernev and Carpenter 2001; Chernev 2007). Thus, if one brand dominates another on some attributes, it should perform less well on others. In the absence of a market-efficiency cue (such as price information), the missing attribute should assume the net valence of the explicit attributes based on the logic of evaluative consistency. However, in the presence of a market-efficiency cue (such as when two brands are comparable in price), a compensatory inference should obtain. Specifically, if one brand is superior to another on the explicitly stated attributes but both are priced equivalently, market efficiency would lead consumers to infer that the inferior brand is better on missing or unstated attributes (Chernev and Carpenter 2001).

The market-efficiency cue reverses the logic of evaluative consistency, ceteris paribus. The question of interest is whether such a reversal will be observed when the marketefficiency cue and the influence of visual design lead to contradictory outcomes. If visual design exerts an exceptionally powerful influence and dominates evaluative consistency, it could also override compensatory inferences, leading consumers to conclude that products with superior aesthetics also perform better on unstated functional attributes, regardless of market-efficiency cues. However, with research showing that, given sufficient cognitive resources, consumers can reason about product attribute levels and make judgments at odds with a visual superiority effect (Hoegg et al. 2010), it is plausible that a strong marketplace cue will nullify any aesthetic influence; therefore, consumers will make compensatory inferences.

H2A: The influence of aesthetic design will override marketplace cues as a basis for inference.

H2B: The influence of aesthetic design will be nullified by marketplace cues as a basis for inference.

We report five studies that investigate the impact of product aesthetics on inference making. Hypotheses $1 \mathrm{~A}$ and $1 \mathrm{~B}$ are examined in studies $1,2 \mathrm{~A}$, and $2 \mathrm{~B}$. Hypotheses $2 \mathrm{~A}$ and $2 \mathrm{~B}$ are examined in studies $2 \mathrm{C}$ and 3 , where the effect of aesthetics on to-be-inferred attributes is examined in the context of competitive markets. In all studies, we present participants with attribute information for two brands and 
elicit an inference about a missing attribute. We examine the influence of aesthetics by including attractive and unattractive product designs and pairing them with inferior or superior functional attributes. Our operationalization of aesthetics overlaps with studies that have construed product attractiveness at a Gestalt level rather than based on particular design characteristics (Orth and Malkewitz 2008; Homburg et al. 2015).

\section{STUDY 1: EVALUATIVE CONSISTENCY}

Study 1 tests hypothesis $1 \mathrm{~A}$ versus hypothesis $1 \mathrm{~B}$ by examining the effect of superior aesthetics on inference making when the superiority of the visual design contradicts informative verbal information. To investigate the effect of aesthetics, we compare the inferences made when explicitly provided information is contradicted by a product image versus when it is contradicted by a verbal (functional) attribute. If aesthetics exert no undue influence, a null effect of information type should be observed. The dependent variable consisted of an inference about a missing attribute.

\section{Participants and Experimental Design}

A total of 227 undergraduate students were randomly assigned to cells of a 2 (information type: verbal vs. visual) $\times 2$ (attribute valence: inconsistent vs. consistent) betweensubjects design. To ensure that the results are not idiosyncratic to a particular attribute, an attribute-replicate manipulation was also included (see below).

\section{Stimuli}

The stimulus consisted of two fictitious smartphone brands, with one brand (brand S) being objectively superior to the other (brand I) based strictly on the number of dimensions on which the brand excelled. Specifically, information about three product attributes was provided, with brand $S$ always superior on at least two of them. Of the three attributes, two were functional attributes that were held constant across conditions. The third attribute varied by condition. In the verbal information conditions, the third attribute referred to another functional attribute (connectivity); in the visual information conditions, the third "attribute" was an image of each smartphone. A fourth attribute was listed, but the values were missing for both brands. Participants were asked to infer the missing value for each brand based on the information provided. For one replicate, the constant attributes were processing speed and memory, and the missing attribute was camera megapixels; for the other replicate, the constant attributes were memory and megapixels, and the miss- ing attribute was processing speed (see app. A for the stimuli; apps. A-G are available online).

To examine how aesthetics influence missing-attribute inferences, the consistency of the valence of the third attribute was manipulated. For participants in the consistentvalence conditions, brand $S$ was superior to brand I on the third attribute; that is, the third attribute was evaluatively consistent with the first two attributes, and therefore brand $S$ outperformed brand I on all three attributes. For participants in the inconsistent-valence conditions, brand $S$ was inferior to brand I on the third attribute, thereby rendering brand S superior overall but not completely dominant.

The verbal attributes were selected in a manner that (1) reflected their widely accepted importance to phone assessment and (2) ensured the missing attribute (megapixels or processing speed) could not be discerned from a visual inspection of the images. Pretests showed that the images differed in attractiveness as intended (7.53 vs. 3.67 on a 9-point scale ranging from $1=$ very unattractive to $9=$ very attractive; $t(29)=10.35, p<.001)$.

\section{Procedure}

Participants imagined a scenario in which they were buying a smartphone for a friend and were considering two brands currently on the market. They were informed that they would see information about the processor speed and memory (or, in the other replicate, camera megapixels and memory) of each brand. Participants in the verbal information conditions were also told they would see the connectivity speed of each brand; participants in the visual information conditions were told they would see images of the phones. Participants were then told that they would be asked a question about camera megapixels (or processor speed) on the next page. Participants were provided with an attribute range that was representative of the market at the time (5.5-8.0 megapixels for camera or $0.5-2.5 \mathrm{GHz}$ for processor speed). Participants were asked to correctly report the range before proceeding. The information about brand $S$ and brand I was then provided and participants were asked to provide an estimate of the camera megapixels (or processor speed) for each brand.

\section{Results and Discussion}

Given the different scales for the missing attribute across replicates, the dependent measure consisted of the proportion of participants who assigned a higher - or more favorable - inferred value of the missing attribute to the inferior brand I. The replicate factor did not produce any significant 
effects (all Wald $<1$ ), indicating robustness of the results and therefore will not be discussed further.

A binary logistic regression showed significant effects of information type (Wald $=3.95, p=.047$ ) and valence (Wald $=5.77, p=.016$ ) as well as a significant interaction (Wald $=9.06, p=.003$ ). Specifically, in the consistentvalence conditions, the proportion favoring the inferior brand (brand I) was significantly lower in the visualinformation condition than in the verbal-information condition $\left(P_{\text {verbal }}=17.9 \%\right.$ vs. $P_{\text {visual }}=3.6 \%$, Bonferroni adjusted $p=.030)$; however, in the inconsistent-valence conditions, the proportion favoring the inferior brand was significantly higher in the visual-information condition than in the verbal-information condition $\left(P_{\text {verbal }}=34.5 \%\right.$ vs. $P_{\text {visual }}=$ $56.9 \%$, Bonferroni adjusted $p=.031$ ). (Multinomial logistic analyses, which took the ties between the brands into consideration, showed consistent results and are reported in app. B.)

Study 1 demonstrated the influence of product aesthetics on inference making. In support of hypothesis $1 \mathrm{~A}$, when a third attribute contradicted two functional attributes, inferences about a missing attribute varied depending on whether the third attribute consisted of visual design or another functional attribute. As expected, when the contradicting third attribute was a functional attribute, consumers made inferences in favor of the otherwise superior brand. In contrast, and counter to an evaluative-consistency effect, a more attractive visual design encouraged participants to make inferences that favored the otherwise inferior brand. It is important to reiterate that evaluative consistency provides a compelling basis for inference. The divergent pattern between the verbal and visual conditions is telling. Aesthetics appear to bias consumers in a way that a verbal attribute does not.

An issue that clouds this interpretation concerns the perceived newness of the competing brands as conveyed by their visual images. Participants may have inferred a superior level of the missing attribute in the visual-inconsistent condition because they perceived it to be a newer offering. To address this possibility, we conducted a within-subject post-test of perceived newness for each visual image (attractive and unattractive) and each level of connectivity (3G and $4 G$ ), which was the attribute that substituted for the images in the verbal conditions. Results showed a significant interaction of attribute type and attribute level $(F(1,24)=$ $14.66 ; p=.001)$. Whereas the phone with a more attractive image was viewed as somewhat newer than one with an unattractive image (4.32 vs. 3.60 on a 7 -point scale; $p<.05$ ), the $4 \mathrm{G}$ phone was perceived as far newer than the $3 \mathrm{G}$ phone ( 4.88 vs. $2.88 ; p<.001$ ). Thus, perceived newness cannot account for the divergent pattern in inferred values between the visual- and verbal-information conditions. Nonetheless, the concern is a legitimate one in a more general sense. The remaining studies control for perceived newness either through pretests of the stimuli or explicit instructions to the participants.

\section{STUDY 2A: FIELD EXPERIMENT}

Before probing additional parameters of the influence of aesthetics, the robustness of the previous findings was examined in a field study with real products and consequential consumer outcomes. Moreover, the present study helps to rule out a visual-dominance explanation by employing visual brand representations across conditions. Specifically, actual products were used in the experimental condition, whereas stylized sketches of the products were used in the control condition.

\section{Participants and Experimental Design}

The study had two conditions: low aesthetic differences (sketches of the products) versus high aesthetic differences (actual physical products). A total of 74 business school students and staff participated. The study was run over the course of three separate days: data for the low-aestheticdifferences condition were collected on the first day, and data for the high-aesthetic-differences condition were collected on the second and third days due to low foot traffic.

\section{Stimuli}

Two models of Bosch electric kettles were selected such that both were the same brand, had a handle and a start button, had the same water capacity, and had hashmarks to indicate fill levels. Importantly, the temperature settings switch (the attribute serving as the missing functional attribute) was located on the base of the kettle. The base was removed so the number of temperature settings could not be determined from a visual inspection.

Across conditions, one brand was objectively superior (brand S) and one was objectively inferior (brand I) based on two functional attributes: boil time and hold time. In the low-aesthetic-differences condition, the two kettle images were processed using software that yielded a detailed black and white, stylized drawing of the product that reduced the aesthetic appeal while still allowing for the general design (e.g., shape, size) and specific visual features (e.g., handle, water fill) to be easily discernible (see app. C). These 
sketches were printed on thick boards and mounted on stands. Each sketch was printed to be exactly the height and width of the corresponding kettle. In the high-aestheticdifferences condition, the two actual kettles were used. All participants received inconsistent-valence information, meaning that brand I was superior to brand $S$ on the visual design (brand $S$ was superior to brand I on functional attributes).

Boil time and hold time were the verbally presented functional attributes, and the number of temperature settings was the missing functional attribute. Participants had to determine whether to form inferences about the superiority of the missing attribute from either the kettle with the superior visual design or from the kettle with superior stated functional attributes. Given the results of study 1 , we expect that when aesthetic differences are reduced (i.e., lowaesthetic-differences condition), people will use the stated functional attributes to form inferences, but when aesthetic differentiation is clear (i.e., high-aesthetic-differences condition) people will use aesthetic design to form inferences.

A pretest of 85 people was run on Prolific Academic, which is composed of primarily UK participants, to be consistent with the subject pool in the main study. Unprocessed photos of each kettle against a solid background were used in place of actual products in this pretest. Participants saw one of the four visual stimuli: an unprocessed photo of a superior- or inferior-looking kettle, or a sketch version of the same superior- or inferior-looking kettle. Participants then rated the attractiveness of the kettle, "How physically attractive (beautiful) is this tea kettle?" ( 1 = extremely unattractive, 7 = extremely attractive) and the newness of the kettle, "How new is this tea kettle?" ( 1 = extremely old, $7=$ extremely new). For those who saw an unprocessed photo, there was no difference in newness perceptions ( $M_{\text {superior }}=$ 5.68 vs. $M_{\text {inferior }}=5.72 ; t<1$ ) but a significant difference in attractiveness perceptions $\left(M_{\text {superior }}=4.68\right.$ vs. $M_{\text {inferior }}=$ 3.92; $t(45)=2.25, p=.029)$. For those who saw a sketch, there was no difference in ratings of newness $\left(M_{\text {superior }}=\right.$ 4.53 vs. $\left.M_{\text {inferior }}=5.26 ; t(36)=1.42, p=.164\right)$ or attractiveness $\left(M_{\text {superior }}=3.84\right.$ vs. $\left.M_{\text {inferior }}=3.89 ; t<1\right)$.

\section{Procedure}

A table was set up around various locations in the business school of University of Oxford advertising a raffle for a $£ 50$ gift card. People were told that they had to guess which of the two kettles had more temperature settings (i.e., missing functional attribute); if they guessed correctly, they would be entered to win the raffle. Additionally, they were asked to indicate which of the two kettles they would want as an additional prize if they won the raffle. This question was asked to determine consequential downstream behavior, as participants would actually receive the kettle. Participants in the low-aesthetic-differences condition saw the two sketches of kettles standing on the table. Accompanying the printed sketches were two product tags designed to look like retail product information labels. Participants in the high-aesthetic-differences condition saw the same information labels, but the actual kettles were sitting on the table. In both conditions, the two kettles were labeled either "A" or "B." Participants responded to the two questions on a slip of paper by circling the corresponding letter and provided their email for entry into the draw. In actuality, all participants were entered to win, and one person was randomly chosen to receive a $£ 50$ Amazon gift card and their selected kettle.

\section{Results and Discussion}

For both the missing functional attribute judgment and the product selection, the choices were tallied. Supporting hypothesis $1 \mathrm{~A}$ over hypothesis $1 \mathrm{~B}$ for the missing functional attribute, $58.0 \%$ of people in the low-aesthetic-differences condition selected brand $S$ as superior compared to only $29.4 \%$ in the high-aesthetic-differences condition $\left(\chi^{2}=6.65\right.$, $p=.010)$. When selecting which product they would like to own, $60 \%$ of people in the low-aesthetic-differences condition selected brand S, whereas only $23.5 \%$ selected it when the actual kettles were present $\left(\chi^{2}=10.87, p<.001\right)$. Participants in the low-aesthetic-differences condition favored the kettle with the superior functional attributes. However, when the products were present and aesthetic design was readily apparent, participants favored the more attractive but functionally inferior kettle both in terms of the missing attribute inference and product selection.

Taken together, studies 1 and 2A provide strong support for hypothesis $1 \mathrm{~A}$. Aesthetic design not only exerted a significant influence on inference in the face of a countervailing logic but, in fact, led to reversals in comparison to the case in which aesthetic information was absent or suppressed. The next study examines the possibility that the influence of design is primarily the result of a cognitive process, wherein consumers interpret superiority in design as a signal of brand superiority.

Study 2B: Nonvisual Depiction of Design Superiority. As noted, there is incipient evidence that consumers interpret 
design as a signal of the firm's commitment to quality (Wu et al. 2017). If so, the design signal could be communicated verbally and still produce a bias in favor of superior design. The present study varied the mode in which design is communicated. Whereas some participants saw images of the brands as in study 1 , others saw attractiveness ratings that signaled that one brand was more attractive than the other. Based on the results of study 2A, inferences are likely to only shift in the direction of the more attractive product when the designs are communicated visually.

\section{Participants and Design}

A total of 251 participants recruited from Amazon Mechanical Turk (MTurk; 56.8\% male, median age $=32$ ) were randomly assigned to cells of a 2 (appearance information type: verbal vs. visual) $\times 2$ (attribute consistency: inconsistent vs. consistent) between-subjects design.

\section{Stimuli}

Two sets of cookware were selected as the stimuli. A pretest was conducted to ensure that the sets of cookware differed on attractiveness but not on perceived newness. Based on the pretest results, profiles for brand $S$ and brand I were constructed (see app. D). In all conditions, the profiles consisted of two functional attributes and one appearance attribute. As in study 1, the two functional attributes (nonstick coating and durability) favored brand $S$. The manner in which appearance was communicated varied by condition. In the visual conditions, images of the two brands were presented; in the verbal conditions, information about the attractiveness of the brands was conveyed in the form of a rating (either " 7.9 out of 10 " or " 6.2 out of 10 "). The rating was derived from the attractiveness ratings of the two cookware sets obtained from the pretest. Regardless of the format of the attractiveness information, both the functional attributes and the appearance attribute favored brand $S$ in the consistent conditions, whereas the functional attributes favored brand $S$, but the appearance attribute favored brand I in the inconsistent conditions.

\section{Procedure}

Participants imagined they were buying a set of cookware. They were told that they would see two sets of cookware and would be given information about several attributes: nonstick coating, durability, and appearance. They were also given some information about how to evaluate each feature (e.g., "durability - measured in millimeters of pan thickness, the thicker the pan is, the more durable"). Participants were told that they would be asked a question about an additional feature, the maximum temperature for each set of cookware, which they were told ranged from $400^{\circ} \mathrm{F}$ to $600^{\circ} \mathrm{F}$. Participants were required to remember and correctly report the range of temperatures before proceeding. They were then presented with the two options and, depending on condition, viewed a set of product profiles described in appendix D. Participants were asked to provide an estimate of the missing attribute (maximum temperature) for both brand $\mathrm{S}$ and brand I. To encourage participants to think carefully about their estimates, assigning equal values to both cookware sets was disallowed, but otherwise their estimates were unconstrained.

\section{Results}

A total of 67 people were removed from the analysis, four of whom failed to use the response scale properly. The remainder possessed a minority view of which cookware set was more attractive, reflective of the subjective nature of design. Values for the missing attribute were converted into difference scores by subtracting the response for the functionally inferior brand (brand I) from the response the functionally superior (brand S) brand. Descriptive results are portrayed in figure 1 (with full details available in app. G).

Analysis revealed main effects of attribute consistency $(F(1,180)=18.40, p<.001)$, appearance information type $(F(1,180)=5.13, p=.025)$, and more importantly, a significant interaction $(F(1,180)=5.04, p=.026)$. No effect of appearance type was observed in the consistent conditions

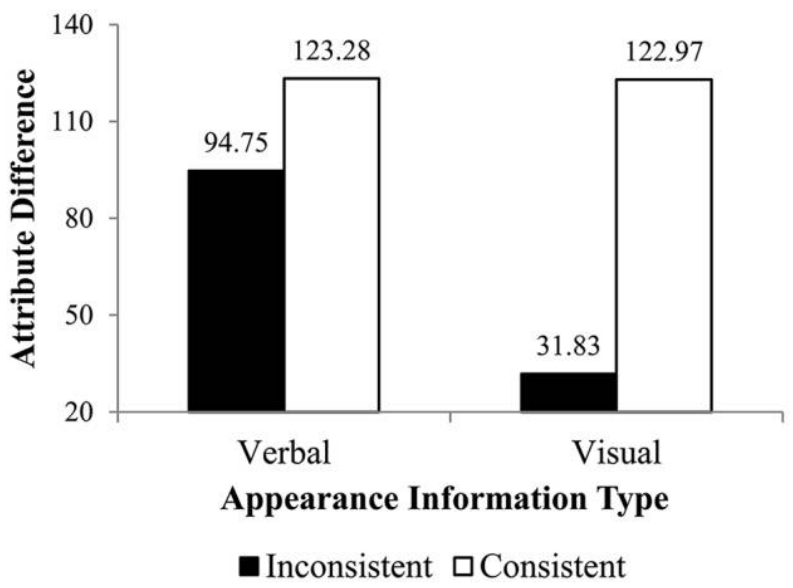

Figure 1. Influence of design on evaluative-consistency inferences (study 2B) 
$\left(M_{\text {verbal }}=123.28\right.$ vs. $\left.M_{\text {visual }}=122.97 ; F<1\right)$, but a significant difference emerged in the inconsistent conditions $\left(M_{\text {verbal }}=94.75\right.$ vs. $M_{\text {visual }}=31.83 ; F(1,180)=9.56, p=$ .002). Thus, when design information was communicated via a numerical rating, participants relied on it very little and provided an estimate of the missing value reflective of evaluative consistency with the other functional attributes. However, when participants viewed the actual designs, the product images had a substantial influence on the estimations of the missing values.

\section{Discussion}

The results of this study support the proposition that the influence of design on inference making is not attributable to strictly cognitive beliefs about the relationship between design and functionality. It is important to note that the effect of the product images was obtained despite confirmation from our pretests that the designs were not diagnostic of the missing attribute. In other words, even though no information about the focal attribute could be discerned from the images, aesthetic design exerted a large influence. Finally, the results are consistent with those of studies 1 and $2 \mathrm{~A}$ regarding support for hypothesis $1 \mathrm{~A}$ over hypothesis $1 \mathrm{~B}$. Once again, despite the presence of other verbal attributes encouraging an inference based on evaluative consistency, visual design exerted a dominating influence on the inference of missing attributes.

Insofar as aesthetic design may be a misleading cue to quality, the implications for consumer welfare are unfavorable. In the next two studies, we examine factors that could lead consumers to be more circumspect.

Study 2 C. If the environment fails to provide reasons to doubt the diagnosticity of aesthetics, consumers will need to be more thoughtful and self-generate reasons for skepticism. Such deliberate reasoning may be prompted by various pieces of information learned from prior experience with the marketplace. For example, consumers may believe that design and functionality naturally trade off in some product categories. Consumers may also take into consideration the firm's reputation, culture, or basis for competitive advantage. In the present study, consumer reaction to design is examined by a simple manipulation of brand name, with one brand implying aesthetic design strength and one brand implying engineering strength. We test whether a brand cue is sufficient to override the influence of aesthetic design (hypothesis 2A vs. 2B).

\section{Participants and Design}

A total of 144 participants were recruited from MTurk $(42.4 \%$ male, median age $=33)$. Each participant was randomly assigned to one of the conditions of a 2 (inconsistent visual information: absent vs. present) $\times 2$ (brand name cue: absent vs. present) between-subjects design.

\section{Stimuli and Procedure}

Laptops served as the product category. Brand $S$ was superior to brand I on the attribute of processing speed but was identical to brand I on the attribute of RAM size. When inconsistent visual information was present, brand $S$ was paired with an inferior image and brand I was paired with a superior image. In the inconsistent-visual-information absent conditions, no product images were presented. The missing attribute was wireless connectivity. To control for perceived newness, participants were asked to imagine that they were considering purchasing a new laptop and had narrowed the choice to two brands that had "recently entered the laptop market with new offerings."

To manipulate brand name, participants in the brandpresent conditions were told that the laptops they would see were from "Brand A by Ultimate Engineering" (assigned to brand S) and "Brand B by Dream Design" (assigned to brand I) whereas the participants in the brand-absent conditions were simply told the laptops were from "brand A" and "brand B." The fonts for the engineering and design brands were also chosen to reinforce the names (see app. E).

The procedure conformed to the preceding studies. After viewing the profiles, participants provided judgments of the quality of the wireless connectivity of the laptops ( 1 = very bad, $10=$ very good $)$ and indicated which laptop they believed provided better wireless connectivity ( 1 = strongly favoring brand A, 10 = strongly favoring brand B).

\section{Results and Discussion}

Nine participants were removed for failing attention check questions (e.g., incorrectly identifying the missing attribute), leaving a final sample size of 135 for analysis. As in prior studies, analyses were first conducted on the difference scores. These analyses revealed significant main effects of visual information $(F(1,131)=18.66, p<.001)$ and brand-name cue $(F(1,131)=8.13, p=.005)$; the interaction was nonsignificant $(F<1)$. Consistent with prior studies, in the brand-name absent conditions, the inconsistent visual image created a favorable bias toward the inferior brand $\left(M_{\text {verbal }}=.94\right.$ vs. $M_{\text {visual }}=-1.03 ; F(1,131)=12.40$, $p<.001)$. More central to the present purpose is the effect 
of the brand cue. Although the brand name cue reduced the bias toward the inferior brand ( $M_{\text {brandpres }}=.36$ vs. $\left.M_{\text {brandabs }}=-1.03 ; F(1,131)=6.02, p=.015\right)$, it did not eliminate it, in that there remained a biased favorability toward the inferior brand $\left(M_{\text {verbal }}=1.80\right.$ vs. $M_{\text {visual }}=.36$; $F(1,131)=6.67, p=.011)$. Analyses of the relativepreference measure were entirely consistent and statistically stronger.

These results again show the biasing effect of aesthetics on inference making while also suggesting that this bias can be attenuated, but not eliminated, by cues that prompt consumers to reflect on the inherent capabilities of the firms that produce attractive versus unattractive designs. Thus, to the detriment of consumer welfare, when design is a misleading cue to quality, we find support for hypothesis $2 \mathrm{~A}$. The final study examines whether attenuation occurs in the context of a different market-based inference (i.e., market efficiency) with a different marketplace cue (i.e., price)

Study 3: Market Efficiency. Recall that the marketefficiency paradigm mimics the evaluative-consistency paradigm when market-efficiency cues are absent. However, in the presence of a market-efficiency cue (e.g., price), when one brand is superior to another on the explicit attributes but both brands are comparable in price, market-efficiency beliefs should lead to the compensatory inference that the brand that is inferior (superior) on the explicit attributes is superior (inferior) on missing attributes. This paradigm extends our investigation to a second basis for inference and examines another avenue by which consumers may avoid the misleading implications of aesthetics.

\section{Participants and Design}

A total of 90 undergraduate students were randomly assigned to the cells of a 2 (visual design information: no image vs. image) $\times 2$ (market efficiency information: no price vs. price) between-subjects design.

\section{Stimuli and Procedure}

Laptop computers again served as the target category. As in study 1, participants imagined a scenario in which they were choosing between two brands of laptop for a friend and would see information about each brand's processor speed and weight. Participants were then told the laptops' memory capacity ranged from 5GB to 9GB. Participants were required to correctly report the range of memory capacity before proceeding. Next, the information about the two attributes (processing speed and weight) was presented. Brand S had more processing speed than brand I. Weight was equal for both laptops. In the price conditions, the price was set at $\$ 799$ for both laptops. This design was adopted in accordance with prior research on compensatory inferences (Chernev 2007). Visual design information was manipulated via the inclusion of product images (see app. F). A pretest established that the two designs differed in attractiveness $(M=7.33$ vs. $M=2.97 ; t(1,29)=9.37$, $p<.001)$.

Memory capacity was the missing attribute. Participants were exposed to condition-appropriate profiles and asked to provide an estimate of the memory capacity for each brand. The estimate was unconstrained.

\section{Results and Discussion}

The no-image conditions provided a baseline for comparison. The no-image/no-price condition should produce inferences favoring the objectively superior brand S. In contrast, prior research suggests that the no-image/price condition should prompt compensatory inferences that favor brand I instead. The critical test of the influence of aesthetics occurs in the image conditions, in which visual design favored the more functional product. If the findings from studies 1 and 2B hold, visual design should negate compensatory thinking and a pattern should be observed that differs from the no-image conditions. Specifically, compensatory inferences should be tempered.

Six participants were removed because they failed to respond on the correct scale. Values for the missing attribute again were converted into difference scores by subtracting the response for the functionally inferior brand (brand I) from the response the functionally superior (brand $\mathrm{S}$ ) brand. Descriptive results are reported in figure 2 (full details available in app. G).

Analysis revealed no effect of market efficiency information $(F(1,80)=1.27, p=.263)$, a main effect of visual design information $(F(1,80)=12.11, p<.001)$, and a significant interaction $(F(1,80)=4.76, p=.032)$. In the noimage conditions, the result was consistent with compensatory inference. Participants who lacked price information estimated that the laptop that was superior on processing speed would also be superior on memory $(M=1.18)$; when price was included, memory estimates shifted significantly toward the laptop that was inferior on processing speed, consistent with the logic of market efficiency $(M=0.07$; $F(1,80)=5.76, p=.019)$. In contrast, when images were presented to participants, the addition of price information 


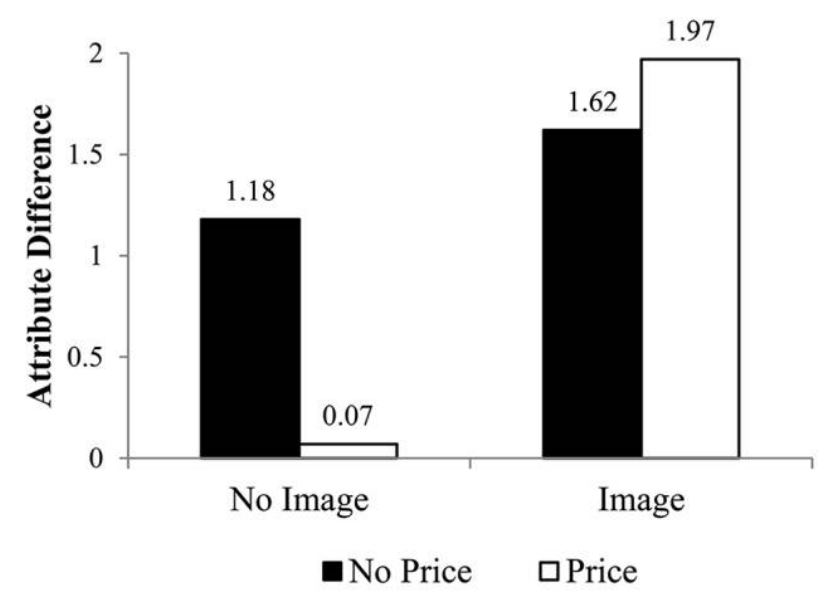

Figure 2. Influence of design on market-efficiency inferences (study 3).

had no effect on memory estimates $\left(M_{\text {noprice }}=1.62\right.$ vs. $M_{\text {price }}=1.97 ; F<1$ ). These results again demonstrate the powerful biasing effect of aesthetics on inference making. Attempts to attenuate this bias with market-efficiency cues (i.e., price) failed to protect the consumer from the undue influence of aesthetics. Thus, the results again support hypothesis $2 \mathrm{~A}$.

\section{CONCLUSION}

Although much is known about how consumers generate inferences about missing attributes based on information about other attributes, less is understood about this process when the attributes include product design. The current research addressed this gap by examining the extent to which aesthetics operates differently from functional attributes in the inference process. Five studies demonstrated that product aesthetics can exert a robust influence on inferences, unlike other verbal attributes. Even for designs lacking information about feature superiority, as was the case in our studies, consumers' judgments of feature performance can be dramatically altered. Our results indicate that aesthetics can override evaluative consistency from explicitly stated attributes which normatively should be far more diagnostic, and furthermore, override other important marketplace cues such as brand reputation and price.

The present findings reflect the importance of everyday aesthetics. None of the products in our studies were unusual in appearance, none were luxury, and all had a primary nonaesthetic purpose. The fact that inferences about the functionality of specific attributes were biased by the products' aesthetics, even in the face of conflicting cues, speaks to the powerful influence of everyday aesthetics. Our results indicate that consumers may be disadvantaged by such biases, choosing products that are objectively suboptimal. Indeed, even in the presence of price information, consumers were swayed by aesthetics.

There is reason for hope, however. Although difficult to reverse, the aesthetic bias can be reduced through branding that signals the value of attributes other than aesthetics even with unknown brands (study $2 \mathrm{C}$ ). A longer, targeted brand campaign may reverse the aesthetic bias. In addition, the influence of design should yield to overwhelming evidence to the contrary. To show that verbal information can prevail, we ran a study within the context of smartphones that manipulated both the presence of brand images and the number of attributes favoring the superior brand. Results showed that in the two-attribute conditions, participants who saw the image estimated the visually superior smartphone (brand I) to have more megapixels than participants who did not see the image $(F(1,63)=14.31, p<.001)$. However, in the three-attribute conditions, participants who saw the image did not significantly differ from participants who did not see the image $(F<1)$.

Several opportunities for additional research are apparent. First, although our studies varied product categories, all focused on functional attributes. It will be important to determine whether the influence of aesthetics on inference occurs similarly for hedonic attributes. Given the affective reaction to visual design identified in the literature, it seems likely that the bias may be even stronger for attributes for which pleasure or enjoyment are paramount. This conjecture is consistent with recent evidence that a cute design increases perceived tastiness, and therefore the overall appeal, of vice products (Schnurr 2019). Second, additional research is needed to attempt to identify ways to increase consumer resistance to design seduction. In particular, consumers may self-correct when they become suspicious of the validity of aesthetics. Warning consumers of the possibility of bias has been shown to reduce the influence of design on product evaluation (Townsend and Shu 2010). Encouraging consumers to question the criteria they are using when formulating their estimates may help to attenuate the bias.

A third area of interest would be to consider whether long term consumer welfare is hampered or helped by the visual design bias. In our studies the visual design bias led participants to make suboptimal inferences, potentially leading to choices of products that underperform. The obvious implication is that such choices would leave consumers worse off. However, the bias could also alter the perception of product 
performance, such that consumers are not less satisfied. This possibility is consistent with Norman's (2004) contention that a beautifully designed product generates positive affect that alters perceived functionality. Much as a strategic label placement can promote social welfare by creating a disposal reluctance (Huang et al. 2019), the aesthetic bias examined in the present research can likewise benefit society if consumers infer that a more attractive product has a smaller environmental footprint. Our results cannot speak to these potential outcomes, but we encourage future research into the possibility that design bias offers some positive implications for consumer welfare.

\section{REFERENCES}

Alba, Joseph W., and J. Wesley Hutchinson (1987), "Dimensions of Consumer Expertise,” Journal of Consumer Research, 13 (4), 411-54.

Bloch, Peter H. (1995), "Seeking the Ideal Form: Product Design and Consumer Response," Journal of Marketing, 59 (3), 16-29.

Broniarczyk, Susan M., and Joseph W. Alba (1994), "The Role of Consumers' Intuitions in Inference Making," Journal of Consumer Research, 21 (3), 393-407.

Chernev, Alexander (2007), "Jack of All Trades or Master of One? Product Differentiation and Compensatory Reasoning in Consumer Choice," Journal of Consumer Research, 33 (4), 430-44.

Chernev, Alexander, and Gregory S. Carpenter (2001), “The Role of Market Efficiency Intuitions in Consumer Choice: A Case of Compensatory Inferences," Journal of Marketing Research, 38 (3), 349-61.

Feldman, Jack M., and John G. Lynch Jr. (1988), Self-Generated Validity and Other Effects of Measurement on Belief, Attitude, Intention, and Behavior," Journal of Applied Psychologv, 73 (3), 421-35.

Hagtvedt, Herik, and Vanessa Patrick (2008), "Art Infusion: The Influence of Visual Art on the Perception and Evaluation of Consumer Products," Journal of Marketing Research, 45 (3), 379-89.

Hoegg, Joandrea, Joseph W. Alba, and Darren W. Dahl (2010), “The Good, the Bad, and the Ugly: Influence of Aesthetics on Product Feature Judgments," Journal of Consumer Psychology, 20 (4), 419-30.

Homburg, Christian, Martin Schwemmle, and Christina Kuehnl (2015), "New Product Design: Concept, Measurement, and Consequences," Journal of Marketing, 79 (3), 41-56.

Huang, Rong, Darren W. Dahl, Shenyu Li, and Qiong Zhou (2019), “The Effect of Packaging Perceptual Cues on Consumer Disposal Behavior of Partially Consumed Products," Journal of the Association for Consumer Research, 4 (4), in this issue.

Jiang, Yuwei, Gerald J. Gorn, Maria Galli, and Amitava Chattopadhyay (2016), "Does Your Company Have the Right Logo? How and Why Circular-and Angular-Logo Shapes Influence Brand Attribute Judgments," Journal of Consumer Research, 42 (5), 709-26.

Kardes, Frank R., Steven S. Posavac, and Maria L. Cronley (2004), "Consumer Inference: A Review of Processes, Bases, and Judgment Contexts," Journal of Consumer Psychology 14 (3), 230-56.
Kardes, Frank R., Steven S. Posavac, and Maria L. Cronley, and Paul M. Herr (2008), "Consumer Inference," in Handbook of Consumer Psychology, ed. Curtis P. Haugtvedt, Paul M. Herr, and Frank R. Kardes, New York: Erlbaum, 165-91.

Labrecque, Lauren, and George Milne (2012), “Exciting Red and Blue: The Importance of Color in Marketing," Journal of the Academv of Marketing Science, 40 (5), 711-27.

Luchs, Michael G., K. Scott Swan, and Marielle E. H. Creusen (2016), "Perspective: A Review of Marketing Research on Product Design with Directions for Future Research," Journal of Product Innovation Management, 33 (3), 320-41.

Luffarelli, Jonathan, Antonios Stamatogiannakis, and Haiyang Yang (2019), "The Visual Asymmetry Effect: An Interplay of Logo Design and Brand Personality on Brand Equity," Journal of Marketing Research, 56 (1), 89-103.

Lynch, John G., Jr., Howard Marmorstein, and Michael F. Weigold (1988), "Choices from Sets Including Remembered Brands: Use of Recalled Attributes and Prior Overall Evaluations," Journal of Consumer Research, 15 (2), 169-84.

Mugge, Ruth, Darren W. Dahl, and Jan P. L. Schoormans (2018), “'What You See, Is What You Get?' Guidelines for Influencing Consumers' Perceptions of Durables through Product Appearance," Journal of Product Innovation Management, 35 (3), 309-29.

Mugge, Ruth, and Jan P. L. Schoormans (2012), "Product Design and Apparent Usability: The Use of Novelty in Product Appearance," Applied Ergonomics, 43 (6), 1081-88.

Norman, Donald A. (2004), Emotional Design: Whv We Love (or Hate) Evervdav Things, New York: Basic Books.

Orth, Ulrich R., and Keven Malkewitz (2008), "Holistic Package Design and Consumer Brand Impressions," Journal of Marketing, 72 (3), 64-81.

Patrick, Vanessa M. (2016), "Everyday Consumer Aesthetics," Current Opinion in Psychologv, 10 (August), 60-64.

Raghubir, Priya, and Eric A. Greenleaf (2006), "Ratios in Proportion: What Should the Shape of the Package Be?" Journal of Marketing, 70 (2), 95107.

Rahinel, Ryan, and Noelle M. Nelson (2016), "When Brand Logos Describe the Environment: Design Instability and the Utility of Safety-Oriented Products," Journal of Consumer Research, 43 (3), 478-96.

Schnurr, Benedikt (2019), "Too Cute to Be Healthy: How Cute Packaging Designs Affect Judgments of Product Tastiness and Healthiness," Journal of the Association for Consumer Research, 4 (4), in this issue.

Townsend, Claudia, and Suzanne B. Shu (2010), "When and How Aesthetics Influences Financial Decisions," Journal of Consumer Psychologv, 20 (4), 452-58.

Veryzer, Robert W., Jr., and J. Wesley Hutchinson (1998), “The Influence of Unity and Prototypicality on Aesthetic Response to New Product Designs," Journal of Consumer Research, 24 (4), 374-94.

Warren, Caleb, and Martin Reimann (2019), "Crazy-Funny-Cool Theory: Divergent Reactions to Unusual Product Designs," Journal of the Association for Consumer Research, 4 (4), in this issue.

Wu, Freeman, Adriana Samper, Andrea C. Morales, and Gavan J. Fitzsimons (2017), "It's Too Pretty to Use! When and How Enhanced Product Aesthetics Discourage Usage and Lower Consumption Enjoyment," Journal of Consumer Research, 44 (3), 651-72. 